\title{
UK Renal Registry 15th Annual Report: Appendix B Definitions and Analysis Criteria
}

\section{B:1 Definition of the incident (take-on) population}

The take-on population is defined as all patients over 18 who started RRT at UK renal centres and did not have a recovery lasting more than 90 days within 90 days of starting RRT.

The treatment timeline is used to define take-on patients as follows.

If a patient has timeline entries from more than one centre then these are all combined and sorted by date. Then, the first treatment entry gives the first date of when they were receiving RRT. This is defined as a 'start date'. However, in the following situations there is evidence that the patient was already receiving RRT before this 'start date' and these people are not classed as take-on patients:

- patients with an initial entry on the timeline of transferred in (modality codes 39 to 69)

- those with an initial entry of transferred out (modality code 38)

- those with an initial treatment of lost to follow up (modality code 95)

- those who had graft acute rejection (modality code 31) and did not have a transplant on the same day

- those with an initial entry of transfer to adult nephrology (modality code 37)

- those with an initial entry of graft functioning (modality code 72)

- those with an initial entry of nephrectomy transplant (modality code 76)

Where none of the above applies, the entry is defined as a take-on (providing there is no recovery of more than 90 days within 90 days of the start date).
C 2013 S. Karger AG, Basel

$1160-2110 / 13 / 1235-0201 \$ 38.00 / 0$

Accessible online at:

www.karger.com/nec
If there is a recovery lasting more than 90 days which begins more then 90 days after starting RRT then the program looks at the modality codes after this date to see if the patient restarted RRT. If they did, then this is classed as another take-on.

For example, a patient may start RRT in 2010, recover and then restart RRT in 2010. Providing that they do not have a recovery lasting more than 90 days within 90 days of start on either occasion, such patients will be counted twice.

See section B:4 'Start of established renal failure' below for information on 'acute' codes such as 81 'acute haemodialysis'.

Provided the Registry received a modality code 36 from the work-up centre, pre-emptive transplants were allocated to the work-up centre and not to the centre where the transplant took place.

Note: patients restarting dialysis after a failed transplant were not counted as a take-on patient.

\section{B:2 Definition of the prevalent population for each year}

The prevalent population for a year is defined as all RRT patients over 18, being treated at centres returning data to the UK Renal Registry (UKRR) for that year and were alive on 31 December of that year. It includes both incident patients for that year and patients who have been on treatment for longer. Note that any patients over 18 who are still being treated at paediatric centres are excluded.

Patients were only included under their primary treatment centre. 
Patients who had transferred out, recovered function, stopped treatment without recovery of function or been lost to follow up before the end of the quarter were excluded.

\section{Further exclusions when analysing quarterly}

biochemistry or BP data

For these analyses, further restrictions were made to the prevalent cohort for each quarter.

Patients who had 'transferred in' to the centre in that particular quarter were excluded.

Patients who had changed treatment modality in that particular quarter were excluded.

Patients who had been on RRT for less than 90 days were excluded.

Note: the length of time on RRT is calculated from the most recent take on date. So if a patient starts, then recovers and then starts again, this second start date is used. Also, for patients who are not defined as take on patients because their start date is unknown (for example, if their first timeline entry is a transfer in code) it is assumed that they have been on RRT for longer than 90 days and they are included for every quarter.

\section{B:3 Statistical definitions}

\section{Death rate calculation}

A death rate per 100 patient years is calculated by counting the number of deaths and dividing by the person years exposed. This includes all patients, including those who died within the first three months of therapy. The person years at risk are calculated by adding, for each patient, the number of days at risk (until they died or transferred out) and dividing by 365 .

\section{Odds ratio}

This is the odds of an event in one group divided by the odds in a reference group. For example, if the event is death (within a certain time) and you are comparing phosphate groups then for phosphate group 1.8 to $2.1 \mathrm{mmol} / \mathrm{L}$ the odds of the event are:

(probability of dying for someone with a phosphate of $1.8-2.1 \mathrm{mmol} / \mathrm{L}$ )

(probability of surviving for someone with a phosphate of $1.8-2.1 \mathrm{mmol} / \mathrm{L}$ )
The odds ratio is then:

(odds of dying if phosphate $1.8-2.1 \mathrm{mmol} / \mathrm{L}$ ) (odds of dying for reference group)

Note that when the event being analysed is death, often the odds ratio would not be used but a 'survival analysis' used instead. This takes into account the time when the event occurs and also allows for censoring (for example if people are lost to follow up). Such an analysis gives hazard ratios (see below) rather than odds ratios.

\section{Hazard function}

The hazard function is the probability of dying in a short time interval, conditional on survival up to that point.

\section{Hazard ratio}

For the same example as above, the hazard ratio is the:

(probability of dying in the next interval for a phosphate of $1.8-2.1 \mathrm{mmol} / \mathrm{L}$ )

(probability of dying in the next interval for a phosphate in the reference range)

\section{Funnel plots}

Percentages achieving RA and other standards are displayed in several ways in the Report. Caterpillar plots show the percentage meeting the targets along with 95\% confidence intervals (CIs) for each centre and overall. Funnel plots show the percentage meeting the target plotted against the size of the centre (the number of people with a measurement). 'Funnels' are plotted around the average percentage meeting the target. Any centres which fall outwith the funnels are significantly different from the average. The funnel shape of the limits reflects the fact that for smaller centres, for which the percentage meeting the target is less reliably estimated, a greater observed difference from the average is required for it to be statistically significantly different.

In survival analysis the funnel plot methodology is similar except that the funnel plots show the percentage survival plotted against the size of the centre (the number of patients in the cohort) and 'funnels' are plotted around the average survival. Survival for any centres falling outside the $95 \%$ confidence intervals is therefore significantly different from the average survival. 
B:4 General and modality definitions

Definitions of analysis quarters

\begin{tabular}{ll}
\hline Quarter & Dates \\
\hline 1 & 1 January-31 March \\
2 & 1 April-30 June \\
3 & 1 July-30 September \\
4 & 1 October-31 December \\
\hline
\end{tabular}

The quarterly biochemistry data are extracted from renal centre systems as the last data item stored for that quarter. If the patient treatment modality was haemodialysis, the software will try to select a pre-dialysis value (unless otherwise specified in the data specification).

\section{Home haemodialysis}

Home haemodialysis patients cease to be classed as such if they need longer than two weeks of hospital dialysis when not an in-patient.

\section{Satellite dialysis unit}

A renal satellite unit is defined as a haemodialysis facility that is linked to a main renal centre, is not autonomous for medical decisions and provides chronic outpatient maintenance haemodialysis but with no acute or in-patient nephrology beds on site.

\section{Start of established renal failure}

Established renal failure (also known as end stage renal failure or end stage renal disease) was defined as the date of the first dialysis (or of pre-emptive transplant).

A patient starting RRT on 'chronic' haemodialysis should be entered on the UKRR timeline on the date of the first HD episode.

If a patient started RRT with an episode of acute (or acute-on-chronic) kidney injury in which it was felt that kidney function had potential to recover, then 'acute haemodialysis' (or acute haemofiltration' where appropriate) should be entered on the UKRR timeline. If subsequently it is felt that kidney function is no longer likely to recover, the timeline modality should be changed to 'chronic haemodialysis' at the time when this becomes apparent (accepting that the timing of this change will vary between clinicians). The UKRR will interrogate the timeline of patients starting 'chronic' RRT and if there is evidence of recent 'acute' RRT, will backdate the date of start to the first episode of 'acute' RRT provided there has been less than 90 days recovery of kidney function between acute and chronic episodes.
If a patient was started on dialysis and dialysis was temporarily stopped for less than 90 days for any reason (including access failure and awaiting the formation of further access), the date of start of renal replacement therapy (RRT) in UKRR analyses remained the date of first dialysis.

The date of start of peritoneal dialysis is defined as the date of first PD fluid exchange given with the intention of causing solute or fluid clearance. This is in contrast with a flush solely for confirming or maintaining PD catheter patency. In general, exchanges which are part of $\mathrm{PD}$ training should be considered as the start of PD (unless earlier exchanges have already been given). However, if it is not planned that the patient starts therapy until a later date, exchanges as part of PD training need not necessarily be considered the start of RRT.

\section{Change of modality from $P D$ to $H D$}

Sites are requested to $\log$ in their timeline changes from $\mathrm{PD}$ to $\mathrm{HD}$ if the modality switch is for longer than 30 days.

\section{Date first seen by a nephrologist}

This is the date the patient first attended clinic or was an inpatient under the care of a dialysing nephrologist (which ever is the earlier). If a patient transfers into a renal centre from another renal centre then this date should be left blank by the new renal centre.

\section{Date of CKD5}

When a patient has two eGFRs recorded as $<15 \mathrm{ml} /$ $\min / 1.73 \mathrm{~m}^{2}$ over a time period of greater than three months apart without an intervening eGFR $>15$, then the earlier of these two dates is defined as the date the patient reached CKD5.

If the patient dies or goes onto RRT within the three month period of eGFR reaching $<15$, then the date of eGFR $<15$ is still the date of CKD5.

\section{B:5 Comorbidity definitions}

Angina

History of chest pain on exercise with or without ECG changes, ETT, radionucleotide imaging or angiography.

Previous MI within last 3 months

Detection of rise and/or fall of a biomarker (CK, CK-MB or Troponin) with at least one value above the 
99th percentile together with evidence of myocardial ischaemia with at least one of either:

(a) ischaemic symptoms,

(b) ECG changes indicative of new ischaemia (new ST-T changes or new left bundle branch block),

(c) development of pathological Q waves,

(d) imaging evidence of new loss of viable myocardium or new regional wall motion abnormality.

This definition is from the European Society of Cardiology and American College of Cardiology.

Previous $M I>3$ months ago

Any previous MI at least 3 months prior to start of renal replacement therapy.

\section{Previous $C A B G$ or coronary angioplasty}

Previous episode of heart failure

Whether or not due to fluid overload.

\section{Cerebrovascular disease}

Any history of strokes (whatever cause) and including transient ischaemic attacks caused by carotid disease.

Diabetes (not causing ESRF)

This includes diet controlled diabetics.

\section{Chronic obstructive pulmonary disease}

Chronic obstructive pulmonary disease (COPD) is characterised by airflow obstruction. The airflow obstruction is usually progressive, not fully reversible and does not change markedly over several months.

- Airflow obstruction is defined as a reduced FEV1 (forced expiratory volume in 1 second) and a reduced FEV1/FVC ratio (where FVC is forced vital capacity), such that FEV1 is less than $80 \%$ predicted and FEV1/FVC is less than 0.7 .
- The airflow obstruction is due to a combination of airway and parenchymal damage.

- The damage is the result of chronic inflammation that differs from that seen in asthma and which is usually the result of tobacco smoke.

There is no single diagnostic test for COPD. Making a diagnosis relies on clinical judgement based on a combination of history, (exertional breathlessness, chronic cough, regular sputum production, frequent winter 'bronchitis', wheeze) physical examination and confirmation of the presence of airflow obstruction using spirometry, (source: British Thoracic Society guidelines).

\section{Liver Disease}

Persistent enzyme evidence of hepatic dysfunction or biospy evidence or HbeAg or hepatitis $\mathrm{C}$ antigen (polymerase chain reaction) positive serology.

\section{Malignancy}

Defined as any history of malignancy (even if curative) e.g. removal of melanoma, excludes basal cell carcinoma.

Claudication

Current claudication based on a history, with or without Doppler or angiographic evidence.

Ischaemic/neuropathic ulcers

Current presence of these ulcers.

Angioplasty, stenting, vascular graft (all non coronary)

This category now includes vascular grafts (e.g. aortic bifurcation graft) and renal artery stents.

\section{Amputation for peripheral vascular disease}

\section{Smoking}

Current smoker or history of smoking within the last year. 